\title{
IMPORTANCIA CULTURAL DE VEGETACIÓN ARBÓREA EN SISTEMAS GANADEROS DEL MUNICIPIO DE SAN VICENTE DEL CAGUÁN, COLOMBIA
}

\section{CULTURAL SIGNIFICANCE OF ARBOREOUS VEGETATION IN LIVESTOCK SYSTEMS OF THE MUNICIPALITY OF SAN VICENTE DEL CAGUAN, COLOMBIA}

\author{
Yimi Katherine Ángel Sánchez ${ }^{1}$, Maggy Ennid Pimentel Tapia ${ }^{2}$, Juan Carlos Suárez Salazar ${ }^{3}$
}

\begin{abstract}
${ }^{1}$ Ingeniero Agroecólogo, Magister en Agroforestería, Profesor Facultad de Ciencias agropecuarias. Universidad de la Amazonia, Sede Principal, calle 17 Diag. 17 Cra. 3F, Barrio Porvenir, Florencia-Caquetá, e-mail: y.angel@udla.edu.co; ${ }^{2}$ Ingeniero Agroecólogo, Facultad de Ingeniería. Sede Principal, calle 17 Diag. 17 Cra.3F, Barrio Porvenir, Florencia-Caquetá, e-mail: madgypimentel@gmail.com; ${ }^{3}$ Ingeniero Agroecólogo, M.Sc. Agroforestería Tropical, Profesor Facultad de Ingeniería, Programa Ingeniería Agroecológica. Universidad de la Amazonia, Sede Principal, calle 17 Diag. 17 Cra. 3F, Barrio Porvenir, Florencia-Caquetá, e-mail: ju.suarez@udla.edu.co
\end{abstract}

Rev. U.D.C.A Act. \& Div. Cient. 20(2): 393-401, Julio-Diciembre. 2017

\section{RESUMEN}

El conocimiento local juega un papel importante en la identificación de especies arbóreas, que contribuyen a la formación de alternativas para sistemas ganaderos, en función a la conservación, la protección del ambiente y la economía de los productores. En la actualidad, se hace necesario conocer alternativas y beneficios para afrontar la variabilidad del clima, que afecta la producción ganadera. Es por ello, que el conocimiento de los productores ganaderos incide sobre la percepción y la toma de decisiones. En este sentido, el objetivo de este estudio fue determinar la importancia cultural de especies arbóreas en los sistemas ganaderos, por medio del conocimiento local. Se realizaron 57 encuestas, para el desarrollo de la metodología de índice de importancia cultural de las especies arbóreas. Se encontró, que las especies para sombra, son las de mayor preferencia; el segundo uso más frecuente es la implementación de árboles para la mitigación y la adaptación al cambio climático y, por último, especies de usos agrícolas. Además, se reconoció que las especies con mayor índice de importancia cultural (IIC) e intensidad de uso (IU) fue Zygia longifolia, con 3,21 IIC y 60\% IU, respectivamente. Asimismo, se reconoció que el uso de especies para sombra son las de mayor predominancia, con 132 menciones y una diferencia significativa de 24 menciones, para cercas vivas. Este estudio permitió reconocer el conocimiento de los productores sobre el valor y el uso de las especies arbóreas y forrajeras, para determinar las especies con mayor importancia cultural.
Palabras clave: Árboles, valor de uso, frecuencias de mención, intensidad de uso.

\section{SUMMARY}

Local knowledge plays an important role in the identification of tree species that contribute to the formation of alternatives to livestock systems in terms of conservation, protection of the environment and producers' economies. At present, it is necessary to know alternatives and benefits to face the variability of the climate that affects livestock production. This is why the knowledge of livestock producers affects the perception and decision making. In this sense, the objective was to determine the cultural importance of tree species in livestock systems through local knowledge. 57 surveys were carried out for the development of the methodology of index of cultural importance of the tree species. It was found that the species for shade are the most preferred; the second most frequent use is the implementation of trees for the mitigation and adaptation to the climatic change and last species of agricultural uses. In addition, it was recognized that the species with the highest cultural importance index (CIU) and intensity of use (UI) were Zygia longifolia with 3.21 IIC and $60 \%$ IU, respectively. Also, it was recognized that the use of species for shade are the most predominant with 132 mentions and a significant difference of 24 mentions for live fences. This study allowed to recognize the knowledge of the producers on the value and use of the tree species and fodder species to determine the species with greater cultural importance. 
Key words: Trees, use value, reference frequencies, intensity of use.

\section{INTRODUCCIÓN}

Los sistemas ganaderos en el mundo son totalmente dependientes de la disponibilidad de los recursos naturales, afectados por el cambio climático (Murgueitio et al. 2014). Este proceso genera un aumento de la variación en la disponibilidad de forraje y trae, como consecuencia, la reducción en la productividad pecuaria (Berrang-Ford et al. 2010; Dulal et al. 2011). Estas condiciones, generan una fuerte necesidad de adoptar alternativas, de sistemas de producción ganadera sostenibles con la conversión ambiental, basada en sistemas silvopastoriles, para hacer frente a estos problemas (Murgueitio et al. 2011).

Estos sistemas de producción son desarrollados en una amplia área del territorio nacional y en diferentes agroecosistemas (Navas, 2010; Smit et al. 2013). Para el departamento del Caquetá, Colombia, son la principal actividad y reglón económico (Fajardo et al. 2014) que se ha desarrollado bajo un sistema tradicional, en donde se transforma el bosque en potrero, con gramas naturales y pastos introducidos, con ausencia de cobertura arbórea (Alarcón \& Tabares, 2007) y, generalmente, sin rotación de potreros, generando problemas, como la degradación del suelo, la contaminación de las aguas y las emisiones de gases con efecto invernadero (Navas, 2007).

Estos cambios son producto del conocimiento y la experiencia que llevan al productor a tomar decisiones, como implementar pasturas y disminuir cobertura arbórea, indicando que se talan áreas de bosque para sembrar pastos y mejorar la alimentación de los animales, ya que la sombra afecta la productividad de los pastos, principalmente aquellos árboles con copas densas (Martínez et al. 2013). De tal manera, las pasturas son una cobertura predominante, que corresponde a pastos limpios y pastos enmalezados, básicamente, utilizados para sustentar una ganadería extensiva (Peña \& Cardona, 2010).

Ante este escenario, es necesaria la búsqueda de modelos de desarrollo y de alternativas de manejo, que trasciendan la realidad actual y redunden en el desarrollo sostenible, generando solución a problemas ambientales y económicos de las comunidades asentadas en la región, además, de mitigar efectos del cambio climático

Según Steinfeld et al. (2009) indican que la producción ganadera tiene en la incorporación de especies arbóreas y arbustos en los pastizales, una opción en la que se conjugan producción, protección y mitigación de los efectos de la radiación solar, ya que los árboles cumplen funciones múltiples, que proveen ingresos económicos y, al mismo tiempo, crean un sistema de producción sustentable, con muchos beneficios ambientales (Harvey et al. 2005). Además, permite aminorar los efectos negativos generados por los sistemas tradicionales, mejorar el bienestar de los animales e incrementar la productividad animal (Navas, 2010).

Al respecto, diferentes autores relacionan la presencia de árboles con diversos bienes y servicios (Couttolenc-Brenis et al. 2005), ya sean maderables, frutales o medicinales, mejoramiento del suelo, artesanal, construcción, forraje, alimento para la fauna silvestre y control biológico (Harmand et al. 2007), reducir la velocidad del viento y contribuir a la conservación del agua y la producción de pastos, en comparación con praderas sin árboles, en condiciones similares (Cuartas et al. 2014), asimismo los árboles, diversifican los ingresos de la finca y del hogar (Bentley et al. 2004).

En este sentido, la selección de los árboles por los productores, se enfatiza en especies nativas, por razones económicas, ambientales y de uso (Garen et al. 2009). Al igual, DeClerck \& Decker (2009) indican que se debe involucrar el nivel de conocimiento de los productores sobre el rol, la dinámica y la preferencia de las especies, ayudando a identificar las especies prioritarias (Lykke et al. 2004); sin embargo, las condiciones culturales, sociales, ambientales y económicas de los productores fijan el tipo de manejo y uso que reciben los árboles.

Es por ello, que los criterios de selección de árboles en los sistemas ganaderos, se determinan por la importancia de los árboles en relación a sus usos y el valor económico en el mercado. Surge, entonces, la pregunta: ¿Cuáles son las especies arbóreas culturalmente más importantes en los sistemas ganaderos del municipio de San Vicente del Caguán?

Según Hoffman \& Gallagher (2007), la forma de obtener información para determinar la importancia cultural de las especies es desarrollando índices que se basan en la asignación de valores de mención para cada uso de las especies. Asimismo, Turner (1988) plantea la necesidad de establecer el nivel de importancia de las especies, de una manera más objetiva y, la cual, evalúa su significado cultural, basándose en una puntuación que se obtiene de la asignación de valores a los factores que contribuyen a su significancia.

Aunado a lo anterior, da Silva et al. (2006) manifiestan que el valor de las especies arbóreas se elabora a partir de un índice de importancia o de valores de uso, por medio del conocimiento local. Por lo anterior, el objetivo del estudio fue 
determinar el conocimiento local e importancia cultural de la vegetación arbórea en sistemas ganaderos, por medio de las percepciones de los usos de árboles, como estrategia de adaptación al cambio climático.

\section{MATERIALES Y MÉTODOS}

Área de estudio: El departamento del Caquetá está localizado al sur del territorio continental colombiano, cerca del $85 \%$ de su territorio pertenece a la unidad de paisaje Llanura Amazónica, ubicada por debajo de los 300msnm. Las temperaturas promedio varían entre $24,8^{\circ} \mathrm{C}$ y $25,9^{\circ} \mathrm{C}$ y la precipitación anual varían entre $3.650 \mathrm{~mm} / \mathrm{año}$. El área de estudio seleccionada fue el municipio de San Vicente de Caguán, ubicada a $02^{\circ} 06^{\prime} 37^{\prime \prime} \mathrm{N} 74^{\circ} 46^{\prime} 07^{\prime \prime} \mathrm{O}$ y a $160 \mathrm{~km}$ al nororiente de Florencia, capital del departamento. Cuenta con un área aproximada de $28.300 \mathrm{~km}^{2}$, de los cuales, $10.703 \mathrm{~km}^{2}$ se encuentran en zona de litigio, con los departamentos del Meta y Guaviare (IGAC, 2003).

El departamento del Caquetá cuenta con el primer hato bovino de la amazonia colombiana y el quinto, a nivel de departamentos, demostrando, en el inventario de bovinos, de 1.304 .403 reses, ubicadas en 12.726 predios, lo que convierte esta actividad agropecuaria, en una de las mayores generadoras de empleo, en los últimos años (Medina et al. 2016). Bajo este contexto, el municipio de San Vicente de Caguán, se destaca por sus actividades agropecuarias, que ha sido el principal motor económico, que hace de este municipio, el primer productor de ganado, a nivel regional, indicando, que el inventario departamental, se concentra en un 46,3\%, en el municipio de San Vicente del Caguan (para el 2015, concentraba el 49\% del hato) (Torrijos et al. 2017).

Población y recolección de la información: Para estimar el índice de importancia cultural (IIC) de las especies arbóreas, se realizaron entrevista a 57 productores con actividad ganadera, seleccionados al azar, que representan $1,5 \%$ de la población censada, con actividad ganadera.

Dicha entrevista incluyó preguntas sobre: ¿Usted ha implementado especies arbóreas, cuáles y por qué?, ¿Qué especies arbóreas utiliza para proporcionar un mejor sombrío para el ganado?, ¿’por qué estás?, ¿Qué especies son útiles para su producción ganadera, por qué?, ¿Cuáles son las razones para tener árboles en los potreros?, ¿Qué especies, de las que mencionó, considera mejor para la producción animal y por qué?, ¿Conoce usted especies de árboles que no generen beneficios al animal?, ¿Cuáles?, ¿Cuál es la importancia de las especies en los sistemas ganaderos doble propósito?, ¿Cuáles son los diferentes usos de los árboles que están en su producción ganadera? y ¿Cuáles son los usos alternos de las especies que tiene sus potreros?
Posteriormente, se construyó una base de datos compuesta por la presencia-ausencia de las especies de árboles, creada del listado libre producido por el productor y clasificados, de acuerdo a su conocimiento, orden, frecuencia de mención; asimismo, el uso actual, dado por los productores, lo que permitió el análisis de la importancia cultural de la vegetación arbórea.

La información fue personalizada, grabada con la autorización del productor y editada, a través del programa Voice editing standard versión 2.0, del formato de audio a texto, para evitar pérdida de información. La determinación taxonómica de las especies proviene de la consulta de base de datos de los herbarios virtuales de COAH Herbario Amazónico Colombiano (https://www.sinchi.org.co/coah), COL, Herbario Nacional Colombiano, Universidad Nacional de Colombia, Bogotá (http://www.biovirtual.unal.edu.co) y Tropicos ${ }^{\circledR}$ (http://www.tropicos.org).

Validación de los resultados: Para el análisis de la información obtenida sobre la vegetación arbórea, se utilizó en índice de importancia cultural, propuesto por Figueroa S. (2000) y utilizado por López-Toledo \& Valdez (2011) y Suárez et al. (2012), que reconoce el conocimiento local entorno a las especies arbóreas (Jiménez et al. 2007):

$$
\mathbf{l u}=\frac{\text { Número de usos de la sp.x }}{\begin{array}{c}
\text { Número total de usos para } \\
\text { todas las especies }
\end{array}} * 100
$$

$$
\mathbf{F m}=\frac{\begin{array}{c}
\text { Número de menciones de la sp.x para } \\
\text { todos los usos }
\end{array}}{\begin{array}{c}
\text { Número total de menciones de todas } \\
\text { las especies para todos los usos }
\end{array}} * 100
$$

$$
\mathbf{V u}=\frac{\begin{array}{c}
\text { Número de menciones de la sp.x } \\
\text { para un uso }
\end{array}}{\begin{array}{c}
\text { Número total de menciones de todas las } \\
\text { especies para un uso }
\end{array}} * 100
$$

$\mathrm{Vut}_{2}=\sum\left(\mathrm{Vu}_{\mathrm{x}}+\mathrm{Vu}_{\mathrm{y}}+\mathrm{Vu}_{\mathrm{z}}+\ldots \ldots . \mathrm{Vu}_{\mathrm{n}}\right)$

$\mathrm{IIC}_{\mathrm{z}}=\left(\mathrm{Iu}_{\mathrm{z}}+\mathrm{Fm}_{\mathrm{z}}+\mathrm{Vutz}\right) / 300$

Dónde: Intensidad de uso (Iu): Porcentaje de usos en los que aparece una especie; Frecuencia de mención (Fm): Sumatoria de menciones para una especie, todos los usos $\mathrm{y}$ todos los informantes; Valor de uso $\mathrm{x}\left(\mathrm{Vu}_{\mathrm{x}}\right)$ : Porcentaje de usos en los que aparece una especie para un uso determinado. 
Los resultados que se obtuvieron en este estudio, se basaron en frecuencias y en porcentajes, de acuerdo al conocimiento y al uso de cada especie, identificada por los productores.

\section{RESULTADOS Y DISCUSIÓN}

En total, se reportaron 84 especies de árboles y de arbustos forrajeros, asociadas a los sistemas ganaderos, correspondientes a 30 familias botánicas, donde se encontró que la familia Fabaceae presentó 18 especies, seguida por Malvaceae, con cinco; Myrtaceae, con cuatro especies, Lamiaceae y Rutaceae, con tres cada uno y 25 familias, representadas por dos o una especie.

Los resultados coinciden con lo reportado por López-Toledo $\&$ Valdez (2011), quienes es su estudio indican que la familia de las Fabaceae, representa la mayor importancia cultural. De igual forma, los ganaderos reconocen que uno de los factores de valor de esta familia de plantas radica en el uso para alimentación, por ser especies forrajeras. Lo que concuerda con Roman et al. (2011), quienes mencionan su importancia por el contenido de proteína del alimento, con relación a especies no leguminosas.

La especie Zygia longifolia (Fabaceae) representó el mayor IIC, con un $3,21 \%$ y un valor significativo de frecuencia de mención, lo cual, muestra para los productores su importancia, por ser una especie útil para el sombrío del animal, protección de fuentes hídricas y el mejoramiento productivo del ganado. Como lo menciona Cerdán et al. (2012), esta especie se considera beneficiosa, ya que sus raíces protegen contra la erosión, cerca de fuentes de agua (Tabla 1). Además, se reconoce, por los productores, que esta especie arbórea representa el mayor número de usos $(n=7)$, potencial para el sistema ganadero doble propósito.

Asimismo, los ganaderos mencionaron Inga densiflora (Fabaceae), Cedrela odorata (Meliaceae), Vitex cymosa (Lamiaceae), con un 2,75\% de IIC, ocupando el segundo puesto en valor de importancia, aunque no compartan los

Tabla 1. Especies con mayor importancia cultural en San Vicente del Caguán.

\begin{tabular}{|l|l|l|l|l|l|l|l|l|l|}
\hline No. & \multicolumn{1}{|c|}{ Familia } & \multicolumn{1}{|c|}{ Especie } & $\begin{array}{c}\text { Nombre } \\
\text { común }\end{array}$ & $\begin{array}{c}\text { No. } \\
\text { Usos }\end{array}$ & Menc & $\begin{array}{c}\text { Iu } \\
\text { rel }\end{array}$ & $\begin{array}{c}\text { Fm } \\
\text { rel }\end{array}$ & $\begin{array}{c}\text { Vux } \\
\text { rel }\end{array}$ & IIC\% \\
\hline $\mathbf{1}$ & Fabaceae & $\begin{array}{l}\text { Zygia longifolia (Humb. \& } \\
\text { Bonpl. ex Willd.) Britton \& Rose }\end{array}$ & Carbón & 7 & 27 & 3,2 & 4,41 & 0,47 & 3,21 \\
\hline $\mathbf{2}$ & Fabaceae & Inga densiflora Benth & Guamo & 6 & 23 & 2,74 & 3,76 & 0,4 & 2,75 \\
\hline $\mathbf{3}$ & Meliaceae & Cedrela odorata L. & Cedro & 6 & 12 & 2,74 & 1,96 & 0,4 & 2,75 \\
\hline $\mathbf{4}$ & Lamiaceae & Vitex cymosa Bertero ex Spreng. & Aceituno & 6 & 35 & 2,74 & 5,72 & 0,4 & 2,75 \\
\hline $\mathbf{5}$ & Fabaceae & Samanea saman (Jacq.) Merr. & Saman & 5 & 19 & 2,28 & 3,1 & 0,33 & 2,29 \\
\hline $\mathbf{6}$ & Acanthaceae & $\begin{array}{l}\text { Trichanthera gigantea (Bonpl.) } \\
\text { Nees }\end{array}$ & Nacedero & 5 & 7 & 2,28 & 2,45 & 0,33 & 2,29 \\
\hline $\mathbf{7}$ & Malvaceae & $\begin{array}{l}\text { Pachira quinata (Jacq.) WS } \\
\text { Alverson }\end{array}$ & Carrecillo & 5 & 10 & 2,28 & 1,63 & 0,33 & 2,29 \\
\hline $\mathbf{8}$ & Melastomataceae & Miconia elata (Sw.) DC. & Chilco & 5 & 7 & 2,28 & 1,14 & 0,33 & 2,29 \\
\hline $\mathbf{9}$ & Olacaceae & Minquartia guianensis Aubl. & Ahumado & 4 & 8 & 1,83 & 1,31 & 0,27 & 1,83 \\
\hline $\mathbf{1 0}$ & Moraceae & Ficus maxima Mill. & Higuerón & 3 & 6 & 1,37 & 0,98 & 0,2 & 1,38 \\
\hline & & otras 74 especies & & & & & 76,17 \\
\hline
\end{tabular}

Iu rel: intensidad de uso relativa; Fm rel: frecuencia de mención relativa; Vux rel: valor de uso x relativo. 
mismos números de menciones, ya que oscilan entre 23 , 12 y 35, respectivamente y seis usos, para cada especie. La especie de Cedrela odorata es prioritaria en conservación, puesto que los principales problemas son las explotaciones forestales, que la categoriza globalmente vulnerable y nacional en peligro (Cardenas \& Salinas, 2007) (Tabla 1).

De acuerdo con la percepción y el conocimiento local de los ganaderos, las especies arbóreas Cedrela odorata y Vitex cymosa son importantes por el aporte económico al ser maderables, además de contar con otros usos, como su aporte de sombra y mitigación del cambio climático. Según De Foresta et al. (2013), los árboles son importantes para los medios de vida de las poblaciones rurales, sea en la provisión de los servicios ecosistémicos o por los ingresos a las familias y al igual que Lasco et al. (2014), reconocen la importancia de los árboles en la adaptación al cambio climático y su mitigación.

Diversos estudios confirman que los productores conocen los beneficios de las especies y las seleccionan y manejan de acuerdo con sus criterios de valor e importancia, lo que influye en una repoblación de las especies, en áreas degradadas (Sánchez et al. 2005; Martínez et al. 2013). Entre las prioridades de los ganaderos para la selección de especies arbóreas y arbustivas, se destaca la provisión de sombra para el animal y la mitigación al cambio climático, además, de nueve usos catalogados por los productores, como importantes.

Es por ello, que Zygia longifolia es de interés para los ganaderos, para el establecimiento de sombra y prestadoras de bienes y servicios, identificada también como la principal especie, por la forma de su dosel; además, resulta ser la especie con mayor número de frecuencias de mención, $4,41 \%$ y considerada con un $70 \%$ de intensidad de uso, seguida por las especies de Trichantera gigantea (Acanthaceae), 60\%, especie importante por su uso forrajero para la suplementación de alimentación animal (Tabla 2), lo que concuerda con Ricaurte et al. (2014), quienes señalan que esta especie es la principal fuente de alimento para el ganado.

Tabla 2. Especies con mayor intensidad de uso y frecuencia de mención en San Vicente del Caguán.

\begin{tabular}{|c|c|c|c|c|}
\hline No & Especie & $\begin{array}{l}\text { Nombre } \\
\text { común }\end{array}$ & Usos & Iu\% \\
\hline 1 & $\begin{array}{l}\text { Zygia longifolia (Humb. \& Bonpl. } \\
\text { ex Willd.) Britton \& Rose }\end{array}$ & Carbón & $\begin{array}{l}\text { Smb, Sa, Ma, Cfhsa, Smb, Pcv, } \\
\text { Psa. }\end{array}$ & 70 \\
\hline 2 & Inga densiflora Benth & Guamo & Sa, Cv, Cfh, Smb, Alm, Dca & 60 \\
\hline 3 & Cedrela odorata $L$. & Cedro & Sa, Ma, Cfhsa, Smb, Pcv Psa. & 60 \\
\hline 4 & Vitex cymosa Bertero ex Spreng. & Aceituno & Sa, Cv, Smb, Pc, Psa, Ma. & 60 \\
\hline 5 & $\begin{array}{l}\text { Trichanthera gigantea (Bonpl.) } \\
\text { Nees }\end{array}$ & Nacedero & Sa, Cfh, Smb, Psa, Alm. & 50 \\
\hline 6 & Samanea saman (Jacq.) Merr. & Samán & Sa, Smb, Psa, Ma, Dca. & 50 \\
\hline 7 & $\begin{array}{l}\text { Pachira quinata (Jacq.) WS } \\
\text { Alverson }\end{array}$ & Carrecillo & Sa, Smb, Psa, Ma, Pcv. & 50 \\
\hline \multirow[t]{3}{*}{8} & Miconia elata (Sw.) DC. & Chilco & Sa, Cfhsa, Smb, Pcv, Psa. & 50 \\
\hline & 74 especies & & $\begin{array}{l}\text { Cv, Ma, Cfhsa, Smb, Pcv, Psa, } \\
\text { Pc, Pbf, Alm, Dca. }\end{array}$ & \\
\hline & TOTAL & & & 100 \\
\hline
\end{tabular}

*Iu \%: Intensidad de uso; Fm: Frecuencia de mención. * Smb: Sombra; Sa: Siembra de árboles para mitigación al cambio climático; Pc: Especies para cultivos agrícolas; Ma: Maderable; Psa: Especies para siembra de árboles dispersos en el potrero; Alm: Alimentación; Dca: Disminución costo de alimentación; Cfhsa: Conservación de fuentes hídricas con siembra de árboles; Pcv: Especies para cercas vivas. 
Entre los usos mencionados por los ganaderos está la sombra, con el mayor número de menciones, que representa el 26,9\%; la siembra de árboles y los arbustos para mitigación del cambio climático, establecido para el control de temperatura y protección de los rayos directos del sol, generando confort del animal, en un $16,1 \%$; especies para cultivos, en un 15,3\%; siembra de árboles dispersos en el potrero, en 11,2\% y, alimentación, con 10\%. Las especies mencionadas para estos usos fueron Zygia, Vitex cymosa; Cedrela odorata e Inga densiflora (Tabla 3).

Estudios han demostrado que tener árboles y arbustos en los potreros contribuyen a la capacidad de proporcionar diversas fuentes alternas, como sombra, alimento para el ganado, regulación del microclima, mejora la estructura y la fertilidad del suelo y optimiza la eficiencia del uso del agua, entre otros (Lasco et al. 2014; Mbow et al. 2014), además, de la captura de carbono, conservación del agua, aire y biodiversidad (Nair, 2012; Czerepowicz et al. 2012).

Del mismo modo, se menciona que la sombra que proporciona Zygia longifolia, Cedrela odorata, Inga densiflora, Miconia elata (Melastomataceae) y Samanea saman (Fabaceae) debe ser uniforme, para disminuir la presión en el suelo, igual a lo reportado por Cipagauta
\& Andrade (2006), donde identificaron que los árboles dispersos en potreros debe proporcionar sombra de una manera uniforme, para evitar que los animales se concentren en un solo espacio, produciendo cárcavas y deterioro en la pradera; sin embargo, los productores aseguran que, en muchos casos, los árboles de sombra no tienen arreglos ni distancias de siembra regulares y muchos no son aptos para el propósito de sombra.

La siembra de árboles dispersos dentro de los potreros no es una práctica común entre los ganaderos, debido a la falta de la relación costo/beneficio, ya que se incurre en gastos para el mantenimiento en los potreros, como el de cercarlos y protegerlos individualmente, durante su establecimiento.

Por otro lado, Pachira quinata (Malvaceae) es mencionada por su aporte para sombra y, como especie maderable, coincide con el estudio de Hall et al. (2011), donde se consideran maderables de alto valor económico; no obstante, la preferencia de los ganaderos por esta especie arbórea, no se basa únicamente en el aporte de sombra, sino en la propagación, lo que consideran como limitante por las condiciones del suelo no adecuadas, lo que corrobora PérezCordero et al. (2003), señalan que esta especie crecen mejor en los sitios fértiles que infértiles.

Tabla 3. Usos alternos de los principales árboles y arbustos forrajeros en el Municipio de San Vicente del Caguán.

\begin{tabular}{|c|c|c|c|c|c|}
\hline \multirow{2}{*}{ No. } & \multirow{2}{*}{ Clave } & \multirow{2}{*}{ Uso } & \multicolumn{2}{|c|}{ Menciones } & \multirow{2}{*}{ Especies más Usadas } \\
\hline & & & Total & (\%) & \\
\hline 1 & Smb & Sombra & 132 & 26,9 & $\begin{array}{l}\text { Zygia longifolia (Humb. \& Bonpl. ex Willd.) } \\
\text { Britton \& Rose, Cedrela odorata L, Inga } \\
\text { densiflora Benth, Miconia elata (Sw.) DC., } \\
\text { Samanea saman (Jacq.) Merr. }\end{array}$ \\
\hline 2 & Sa & $\begin{array}{l}\text { Siembra de árboles } \\
\text { para mitigación }\end{array}$ & 79 & 16,1 & $\begin{array}{l}\text { Cedrela odorata L., Inga densiflora Benth, } \\
\text { Manihot esculenta Crantz. }\end{array}$ \\
\hline 3 & Pc & $\begin{array}{l}\text { Especies para cultivos } \\
\text { agrícolas }\end{array}$ & 75 & 15,3 & $\begin{array}{l}\text { Manihot esculenta Crantz., Musa paradisiaca } \\
\text { L., Citrus sinensis (L.) Osbeck, Mangifera } \\
\text { indica L. }\end{array}$ \\
\hline 4 & Psa & $\begin{array}{l}\text { Especies para siembra } \\
\text { de árboles dispersos } \\
\text { en el potrero }\end{array}$ & 55 & 11,2 & $\begin{array}{l}\text { Zygia longifolia (Humb. \& Bonpl. ex Willd.) } \\
\text { Britton \& Rose, Cedrela odorata L. Vitex } \\
\text { cymosa Bertero ex Spreng. Trichanthera } \\
\text { gigantea (Bonpl.) Nees }\end{array}$ \\
\hline 5 & Alm & Alimentación & 49 & 10 & $\begin{array}{l}\text { Inga densiflora Benth, Pueraria phaseoloides } \\
\text { (Roxb.) Benth. }\end{array}$ \\
\hline
\end{tabular}


Aunado a lo anterior, los productores del municipio quienes poseen especies forestales afirmaron que su distribución se debe a una propagación natural, a través de la dispersión de semillas por el viento y las aves; a su vez, los ganaderos reconocen que mantener dichas especies genera beneficios para el productor y el sistema ganadero. De igual manera, Ramírez et al. (2010) mencionan los beneficios de los árboles en los potreros como la disponibilidad constante de alimento, sombra, que evitan grandes pérdidas de peso o disminución en la producción de leche y mejoran los ingresos a mediano y largo plazo.

Conflictos de intereses: El manuscrito fue preparado y revisado con la participación de los autores, quienes declaramos que no existe conflicto de intereses que ponga en riesgo la validez de los resultados presentados.

\section{BIBLIOGRAFÍA}

1. AlARCÓN, M.; TABARES, E. 2007. Economía y usos de la biodiversidad: actividad pecuaria. En: Diversidad biológica y cultural del sur de la Amazonia colombiana-Diagnóstico. Ruiz, S.L.; Sánchez, E.; Tabares, E.; Prieto, A.; Arias, J.C., Gómez, R., Castellanos. D.; García, P.; Rodríguez, L. (eds.) Corpoamazonia, Instituto Humboldt, Instituto Sinchi, UAESPNN, Bogotá. P.314-316.

2. BENTLEY, J.W.; BOA, E.; STONEHOUSE, J. 2004. Neighbour trees: Shade, intercropping and cacao in Ecuador. Rev. Human Ecology. 32(2):241-270.

3. BERRANG-FORD, L.; FORD, J.D.; PATERSON, J. 2010. Are we adapting to climate change? Rev. Global Environ Change. 21(1):25-33.

4. CARDENAS, D.; SALINAS, N. 2007. Libro rojo de plantas de Colombia". Especies maderables amenazadas: Primera parte. Serie libros rojos de especies amenazadas de Colombia. Bogotá. Colombia. Instituto Amazónico de Investigaciones Científicas "SINCHI". Ministerio de Ambiente vivienda y desarrollo territorial. 4:234.

5. CERDÁN, C.R.; REBOLLEDO, M.C.; SOTO, G.; RAPIDEL, B.; SINCLAIR, F.L. 2012. Local knowledge of impacts of tree cover on ecosystem services in smallholder coffee production systems. Rev. Agricultural Systems, 110(1):119-130.

6. CIPAGAUTA, M.; ANDRADE, H.J. 2006. Sistemas silvopastoriles, una alternativa para el manejo sostenible de la ganadería en la Amazonía.
Corporación Colombiana de Investigación Agropecuaria. 21p.

7. COUTTOLENC-BRENIS, J.A.; CRUZ, E.; CEDILLO, M.Á.; MUSÁLEM, M. 2005. Uso local y potencial de las especies arbóreas en camarón de Tejeda, Veracruz. Universidad Autónoma Chapingo México. Rev. Chapingo. Serie Ciencias Forestales y del Ambiente. 11(1):45-50.

8. CUIARTAS, C.A.; NARANJO, J.F.; TARAZONA, A.M.; MURGUEITIO, E.; CHARÁ, J.D.; KU, J.; SOLORIO, F.J.; FLORES, M.X.; SOLORIO, B.; BARAHONA, R. 2014. Contribution of intensive silvopastoral systems to animal performance and to adaptation and mitigation of climate change. Rev. Colombiana de Ciencias Pecuarias. 27(2):76-94.

9. CZEREPOWICZ, L.; CASE, B.; DOSCHER, C. 2012. Using satellite image data to estimate aboveground shelterbelt carbon stocks across an agricultural landscape. Rev. Agriculture, Ecosystems \& Environment 156:142-150.

10. DASILVA, V.A.;ANDRADE, L.D.H.C.;DEALBUQUERQUE, U.P. 2006. Revising the Cultural Significance Index: the case of the Fulni-ô in Northeastern Brazil. Rev. Field Methods. 18(1):98-108.

11. DE FORESTA, H.S.E.; TEMU, A.; BOULANGER, D.; FEUILLY, H.; GAUTHIER, M. 2013. Towards the Assessment of Trees Outside Forests: A Thematic Report Prepared in the Framework of the Global Forest Resources Assessment 2010. Ed. D. Taylor. Rome, IT, FAO. (Forest Resources Assessment Working Paper 183). 335p.

12. DeCLERCK, F.; DECKER, M. 2009. Integrando la adaptabilidad al cambio climático través de la biodiversidad. In: Sepúlveda, C.; Ibrahim, M. (eds). Políticas y sistemas de incentivos para el fomento y adopción de buenas prácticas agrícolas como una medida de adaptación al cambio climático en América Central. CATIE, Turrialba, CR. p.23-39.

13. DULAL, H.D.; BRODNIG, G.; SHAH, K.U. 2011. Capital assets and institutional constraints to implementation of greenhouse gas mitigation options in agriculture. Rev. Mitigation and Adaptation Strategies for Global Change. 16(1):1-23.

14. FAJARDO, M.Y.; VARGAS, G.F.; MARÍN, L.A.V. 2014. Costos ambientales y evaluación social en conversión 
de los Sistemas de producción ganadera tradicional al sistema silvopastoril en fincas ganaderas de los municipios de Florencia, Morelia y Belén de los Andaquíes del departamento del Caquetá. Rev. Momentos de Ciencia. (Colombia). 11(1):50-57.

15. FIGUEROA S., M. E. 2000. Uso agroecológico, actual y potencial, de especies arbóreas en una Selva Baja Caducifolia perturbada del suroeste del Estado de México. Tesis de Maestría. Colegio de Posgraduados. Montecillo, Texcoco, México. 120p.

16. GAREN, E.J.; SALTONSTALL, K.; SLUSSER, J.L.; MATHIAS, S.; ASHTON, M.S.; HALL, J.S. 2009. An evaluation of farmers' experiences planting native trees in rural Panama: implications for reforestation with native species in agricultural landscapes. Agroforestry Systems. 76(1):219-236.

17. HALL, J.S.; LOVE, B.E.; GAREN, E.J.; SLUSSER, J.L.; SALTONSTALL, K.; VAN BREUGELA, M.; IBARRAA, D.; BORKB, E.; SPANERB, D.; WISHNIEA, M. 2011. Tree plantations on farms: Evaluating growth and potential for success. Rev. Forest Ecology and Management. 261(10):1675-1683.

18. HARMAND, J.M.; AVILA, H.; DAMBRINE, E.; SKIBA, U.; DE MIGUEL, S.; RENDEROS, R.V.; OLIVER, R.; JIMÉNEZ, F.; BEER, J. 2007. Nitrogen dynamics and soil nitrate retention in a Coffea arabica-Eucalyptus deglupta agroforestry system in Southern Costa Rica. Rev. Biogeochemistry. 85(2):125-139.

19. HARVEY, C.A.; VILLANUEVA, C.; VILLACIS, J.; CHACÓN, M.; MUÑOZ, D.; LÓPEZ, M.; IBRAHIM, M.; GÓMEZ, R.; TAYLOR, R.; MARTÍNEZ, J.; NAVAS, A.; SÁENZ, J.; SÁNCHEZ, D.; MEDINA, A.; VÍLCHEZ, S.; HERNÁNDEZ, B.; PÉREZ, A.; RUIZ, F.; LÓPEZ, F.; LANG. I.; SINCLAIR, F.L. 2005. Contribution of live fences to the ecological integrity of agricultural landscapes. Agriculture Ecosystems \& Environment. 111(1):200-230.

20. HOFFMAN, B.; GALLAGHER, T. 2007. Importance indices in Ethnobotany. Ethnobotany Research \& Applications. Rev. Mexicana de Biodiversidad. 5:201218.

21. INSTITUTO GEOGRÁFICO AGUSTÍN CODAZZI -IGAC-. 2003. Mapa de suelos de Colombia. Departamento Administrativo Nacional de Estadística, DANE Subdirección de Agrología.
22. JIMÉNEZ-FERRER, J.G.; PÉREZ-LÓPEZ, H.; SOTOPINTO, M.L.; NAHED-TORAL, J.; HERNÁNDEZLÓPEZ, L.; CARMONA DE LA TORRE, J. 2007. Livestock, nutritive value and local knowledge of fodder trees in fragment landscapes in Chiapas, Mexico. Rev Interciencia. 32(4):274-280.

23. LASCO, R.D.; DELFINO, R.J.P.; CATACUTAN, D.C.; SIMELTON, E.S.; WILSON, D.M. 2014. Climate risk adaptation by smallholder farmers: the roles of trees and agroforestry. Current Opinion. Environmental Sustainability. 6:83-88.

24. LÓPEZ-TOLEDO, J.F.; VALDEZ, J.I. 2011. Uso de especies arbóreas en una comunidad de la reserva de la Biósfera la Sepultura, Estado de Chiapas. In Bosques y Árboles del Trópico Mexicano: Estructura, crecimiento y usos. P.57-79.

25. LYKKE, A.M.; KRISTENSEN, M.K.; GANABA, S. 2004. Valuation of local use and dynamics of 56 woody species in the Sahel. Biodiversity and Conservation. 13(10):1961-1990.

26. MARTÍNEZ-ENCINO， C.; VILLANUEVA-LÓPEZ， G.; CASANOVA-LUGO, F.2013. Densidad y composición de árboles dispersos en potreros en la Sierra de Tabasco, México. Agrociencia. 47(5):483-496.

27. MBOW, C.; VAN NOORDWIJK, M.; LUEDELING, E.; NEUFELDT, H.; MINANG, P.A.; KOWERO, G. 2014. Agroforestry solutions to address food security and climate change challenges in Africa. Current Opinion. Environmental Sustainability. 6:61-67.

28. MEDINA, Y.C.; CARDOZO, O.L.; SANTOS, F.E. 2016. Comercialización de ganado bovino en pie entorno a la compañía de ferias y mataderos del Caquetá (COFEMA) Florencia-Caquetá. Revista FACCEA. 6(1):81-89.

29. MURGUEITIO, E.; CALLE, Z.; URIBE, F.; CALLE, A.; SOLORIO, B. 2011. Native trees and shrubs for the productive rehabilitation of tropical cattle ranching lands. Rev Forest Ecology and Management. 261(10):1654-1663.

30. MURGUEITIO, E.; CHARÁ, J.; BARAHONA, R.; CUARTAS, C.; NARANJO, J. 2014. Los sistemas silvopastoriles intensivos (SSPi), herramienta de mitigación y adaptación al cambio climático. Rev. Tropical and Subtropical Agroecosystems. 17(3):501 $-507$ 
31. NAIR, P.K.R. 2012. Carbon sequestration studies in agroforestry systems: a reality-check. Rev Agroforestry Systems. 86(2):243-253.

32. NAVAS, A. 2007. Sistemas silvopastoriles para el diseño de fincas ganaderas sostenibles. Rev. Acovez. 37(3):16-20.

33. NAVAS, A. 2010. Importancia de los sistemas silvopastoriles en la reducción del estrés calórico en sistemas de producción ganadera tropical. Rev. Medicina Veterinaria. (19):113-122.

34. PEÑA, C.P.; CARDONA, G. 2010. Dinámica de los suelos amazónicos: Procesos de degradación y alternativas para su recuperación. Instituto Sinchi. Bogotá, Colombia. 122p.

35. PÉREZ-CORDERO, L.D.; KANNINEN, M.; UGALDEARIAS, L.A. 2003. Stand growth scenarios for Bombacopsis quinata plantations in Costa Rica. Forest Ecology and Management. 174(1):345-352.

36. RAMÍREZ-AVILÉS, L.; CASTILLO, C.J.B.; CHAY, C.A.J.; SOLORIO, S.F.J. 2010. Rendimiento y calidad de pasturas tropicales bajo condiciones de sombra. En: Velasco, Z.; Ma, A.; Hernández, G.A.; Pérezgrovas, G.; Sánchez M., B. (eds). Los Forrajes y su Impacto en el Trópico. FMVZ-UNACH. Chiapas, México. P.249-267.

37. RICAURTE, L.F.; WANTZEN, K.M.; AGUDELO, E.; BETANCOURT, B.; JOKELA, J. 2014. Participatory rural appraisal of ecosystem services of wetlands in the Amazonian Piedmont of Colombia: elements for a sustainable management concept. Wetlands ecology and management. 22(4):343-361.

38. ROMAN, M.L.; MORA SANTACRUZ, A.; GALLEGOS, R.A. 2011. Árboles tropicales de uso múltiple en la costa de Jalisco, México. En: Endara Agramont,
Á.R.; Mora Santacruz, A.; Valdez-Hernández J.I. (eds.) Bosques y Árboles del Trópico Mexicano: Estructura, crecimiento y usos. Guadalajara, Jalisco. p.82-106.

39. SÁNCHEZ M. D.; A. HARVEY, C.; A. GRIJALVA, A.; MEDINA, S.; VÍLCHEZ, B.; HERNÁNDEZ. 2005. Diversidad, composición y estructura de la vegetación en un agropaisaje ganadero en Matiguás, Nicaragua. Rev. Biol. Trop. 53(4): 387-414.

40. SMIT, H.H.; MEIJAARD, E.; VAN DER LAAN, C.; MANTEL, S.; BUDIMAN, A.; VERWEIJ, P. 2013. Breaking the link between environmental degradation and oil palm expansion: a method for enabling sustainable oil palm expansion. PloS one, 8(9):e68610.

41. STEINFELD, H.; GERBER, P.; WASSENAAR, T.; CASTEL, V.; ROSALES, M.; DE HAAN, C. 2009. La larga sombra del ganado. Problemas ambientales y soluciones. LEAD - FAO. Viale delle Terme di Caracalla 00153 Roma, Italia. 464p.

42. SUÁREZ, A.; WILLIAMS-LINERA, G.; TREJO, C.; VALDEZ-HERNÁNDEZ, J.I.; CETINA-ALCALÁ, V.M.; VIBRANS, H. 2012. Local knowledge helps select species for forest restoration in a tropical dry forest of central Veracruz, Mexico. Agroforestry systems. 85(1):35-55.

43. TORRIJOS, R.; ESLAVA, F.; BENTRAN, Y. 2017. Nueva ganadería del Caquetá en cifras 2016. Editorial Comité Departamental de Ganaderos del Caquetá. Florencia - Caquetá. Colombia. 40p.

44. TURNER, N.J. 1988. The importance of a Rose: Evaluating the cultural significance of plants in Thompson and Lillooet Interior Salish. American Anthropologist. 90(2):272-290.

Recibido: Julio 29 de 2016

Aceptado: Septiembre 18 de 2017

Cómo citar:

Angel Sánchez, Y.K.; Pimentel Tapia, M.E.; Suárez Salazar, J.C. 2017. Importancia cultural de vegetación arbórea en sistemas ganaderos del municipio de San Vicente del Caguán, Colombia. Rev. U.D.CA Act. \& Div. Cient. 20(2): 393-401. 